\title{
MANIFESTACIONES DE DISCRIMINACIÓN EN CONTRA DE LAS PERSONAS QUE SE RECONOCEN CON ORIENTACIÓN SEXUAL DIVERSA EN LA UNIAJC. CASO INSTITU- CIÓN UNIVERSITARIA ANTONIO JOSÉ CAMACHO
}

\section{Carlos Alberto Sarria Trejos, Lizeth Daniela García Chicho, Dorlaomer Villa Guzmán y Vivian Figueroa} Mejía.

Semillero Trabajo Social

Grupo de Investigación Anudamientos

Institución Universitaria Antonio José Camacho

Recepción: 01/08/2019. Aceptado: 07/10/2019.

Cómo citar este artículo:

Sarria Trejos, C.A., García Chicho, L.D., Villa Guzmán, D. \& Figueroa Mejía, V. (2019). Manifestaciones de discriminación en contra de las personas que se reconocen con orientación sexual diversa en la UNIAJC. Caso Institución Universitaria Antonio José Camacho. Revista Sapientía, 11 (22), 38-51.

\section{RESUMEN}

El artículo pretende evidenciar las manifestaciones de discriminación que se presentan en contra de las personas que se identifican con una orientación sexual diferente a la heterosexual en el contexto universitario. La idea surge a partir del proyecto de grado denominado "Manifestaciones de discriminación que se ejerce en contra de las personas con orientación sexualmente diversa entre los estudiantes de la UNIAJC sede norte jornada diurna", desarrollado en la Institución Universitaria Antonio José Camacho. La investigación fue de tipo comprensivo - explicativo, utilizando complementariedad de métodos con mayor énfasis en los datos cuantitativos. Las manifestaciones de discriminación se observaron con la población de la jornada diurna, a partir de la voz y percepción de los estudiantes. El fenómeno se entiende desde la interacción simbólica, comprendiendo aspectos de la diversidad desde la teoría Queer, desde aquí, se percibe la realidad de la diversidad en la interacción a partir de prácticas establecidas que se sustentan en la cultura patriarcal así, comprender los sentidos que se le da a la diversidad sexual, que llevan a procesos de estigmatización y de discriminación al interior de la Universidad. Se concluye que las manifestaciones de discriminación se centran en el sentido que los jóvenes le dan a las acciones de los pares con orientación sexual diversa, que las manifestaciones de discriminación se presentan en discursos que niegan las expresiones centradas en la conducta pública asumida, que el espacio en el cual se relacionan los individuos influye en los comportamientos frente a la diversidad y en las dinámicas de relación centradas en las prácticas culturales aceptadas de manera formal.

\section{PALABRAS CLAVE}

Discriminación, diversidad sexual, derechos humanos, género, universidad.

\section{ABSTRACT}

This research article aims to demonstrate the manifestations of discrimination faced by people who have experienced sexual orientation different from heterosexual in the university context. This article 
comes from the project called "Manifestations of discrimination shown against people with diverse sexual orientation among the students of the Uniajc north branch in the morning shift" developed in the Insitucion Universitaria antonio José Camacho. The research carried out was a comprehensive-explanatory type, using complementarity methods with emphasis on quantitative data. The manifestations of discrimination were observed within the population of the morning shift, based on the students testimonials. The phenomenon is understood from the symbolic interaction, where the relationships are given due to the interaction based on established practices taken from the patriarchal cultural. In this way to show a clear understanding of the meanings given to sexual diversity. As a result there are several processes of stigmatization and discrimination within the university. The article concludes that the manifestations of discrimination focus on the meaning that young people give to the actions of peers with diverse social orientation.

\section{KEYWORDS}

Discrimination, sexual diversity, human rights, gender, university

\section{INTRODUCCIÓN}

La investigación "Manifestaciones de discriminación que se ejerce en contra de las personas con orientación sexualmente diversa entre los estudiantes de la UNIAJC sede norte jornada diurna" pretende hacer visible en un espacio concreto como lo es la universidad, la discriminación, las percepciones sobre aceptación o negación de la diversidad sexual, el reconocimiento de la universidad como escenario de control, de interacción y de libertad. Las diferentes concepciones que se tienen acerca de las personas con orientación sexual diversa a la heterosexual, tienen implicaciones frente a la forma de construir las relaciones entre los sujetos sociales que comparten un espacio territorio común, a partir de estas percepciones y representaciones los individuos actúan de diversas formas. En un escenario como el universitario, las acciones pueden desarrollarse desde el desconocimiento del otro, desde el invisibilizar la existencia, desde la burla frente al percibido como diverso, desde la discriminación física, verbal y no verbal o simplemente desde la interacción tolerante o el absoluto respeto a la diversidad.

El artículo pretende retomar las investigaciones que han dado fuerza a la reflexión sobre el fenómeno de discriminación en ámbitos universitarios con el fin de hacer una revisión del tema para la compresión de las manifestaciones del fenómeno en instituciones abiertas de palabra a la diversidad. En este sentido, el texto realiza una reflexión teórica desde la teoría Queer, que sostiene que los géneros, las identidades sexuales, y orientación sexual no son inherentes al ser humano, rechaza las ideas de dicciones y roles en la orientación sexual, que, al ser producto de lo social y cultural, estigmatizan las identidades, es decir, que la orientación sexual se concibe como un derecho humano que es transgredido por el orden formalmente establecido. Por su lado, observar desde el interaccionismo simbólico las relaciones que se establecen entre pares diversos en un espacio concreto, entendiendo la interacción simbólica como aquella encargada de comprender el conjunto de símbolos y significados intersubjetivos que se establecen en el marco relacional y que determinan como manifestaciones la discriminación. En este orden de ideas, la discriminación se expresa como la acción percibida, significada frente al proceso de interacción de los estudiantes, de jóvenes que están consolidando y reafirmando su identidad.

El esquema metodológico establece un tipo de investigación comprensiva - explicativa, a partir de la combinación de métodos desde un enfoque de complementariedad, con mayor énfasis en los datos cuantitativos. El artículo en primera instancia realiza una revisión del conjunto de investigaciones que acercan al tema, continua con una descripción teórica y metodológica y presenta los ha- 
llazgos más relevantes para finalmente presentar las conclusiones y recomendaciones.

\section{ACERCAMIENTO AL PROBLEMA: LAS MANIFESTACIONES DE DISCRIMINACIÓN EN CONTRA DE LA ORIENTACIÓN SEXUAL DIVERSA}

La discriminación hacia las personas que se identifican como sexualmente diversas es entendida como una problemática que afecta todos los entornos sociales, teniendo en cuenta que abarca un trato excluyente y de rechazo gracias a los estigmas que se han impuesto a lo largo de la historia en la sociedad, lo cual ha llevado a la construcción de una imagen y una conducta que deben de ser aceptadas y mantenidas frente a los demás.

Hoy en día el tema de la orientación sexual diversa, es abordado desde la opinión pública desde diversos puntos de vista, por un lado, la aceptación de la diversidad, las políticas de respeto, de tolerancia, como también de las prácticas en torno a ver la diversidad como perversión; desde estas posturas, se ha podido develar las formas de discriminación hacia las personas con orientación sexual diversa. Lo que no ha quedado claro para muchos es que la sexualidad es un concepto que está relacionado con la construcción de la vida humana, es un proceso que se da de forma natural en los seres humanos, aunque se da en un contexto socio cultural, es decir, que se somete contextualmente a los procesos de construcción social y cultural establecidos, los cuales determinan cómo deber ser el comportamiento de un hombre y una mujer en cuanto a roles, costumbres y extiende su determinismo hacia la orientación sexual heterosexual, lo cual impacta directamente en la búsqueda de aceptación de los individuos diversos en la sociedad.

La orientación sexual enmarca la atracción sexual y emocional que una persona puede tener por otra, sin tener en cuenta el género ni el sexo biológico; también vincula prácticas de comportamiento que se adoptan a partir de definir la orientación sexual, lo cual termina de constituirse en parte de su identidad. Hoy en día se han identificado diferentes orientaciones, que determinan un avance en términos de la acepción de libertad en los seres humanos y otras formas de reconocer que no solo la heterosexualidad es la única forma de relación y de orientación. Con diversos matices se puede ser gay, homosexual, lesbiana, bisexual o heterosexual, sin embargo, al asumirlas en un sistema patriarcal, homofóbico e intolerante, que valora como única definición la orientación heterosexual, excluye y determina acciones de discriminación contra personas que, o se determinan como diversos, o representan características que los vinculan a grupos de orientación sexual diversa.

Cuando una persona viene al mundo, inicia el proceso de construcción de su desarrollo físico, emocional, social y cultural, y son las instituciones las primeras intermediarias que influyen en estos procesos, inculcando creencias y prácticas en el proceso de socialización, que impulsan a los individuos a ejercer roles determinados como válidos en la sociedad o, en su defecto, a ir generando diferencias y resistencias frente a estos roles determinados. En ese escenario, la socialización sobre orientación sexual prevista está determinada por el proceso histórico que ha definido culturalmente los prototipos de hombre y mujer, en otros términos, la heteronormatividad como regla única de referencia que se impone en sistemas de corte patriarcal. La gran pregunta tiene que ver con quien identifica o se identifica por fuera de este sistema de roles. La diversidad sexual que no ha sido reconocida históricamente como parte de la complejidad humana ha sido desvirtuada y entendida como un fenómeno anormal que impide al individuo ser reconocido humanamente como sujeto de derechos, a concebirlo como ciudadano de segunda o tercera categoría, o como ser enfermo y no válido. Y ¿qué es la diversidad sexual? Pues bien, esta es definida como el reconocimiento que tiene una persona de sí misma, lo cual involucra su forma de pensar, sus gustos y preferencias sexuales (Jaramillo \& Morales, 2018). 


\section{¿Qué se entiende por discriminación?}

La discriminación es entendida como aquellos comportamientos que naturalizan la no aceptación de quien es percibido como diferente y que se acompañan de prejuicios, discursos y prácticas sociales centradas en el desconocimiento y la exclusión. Para Sevilla-González (2012), "La actitud ni siquiera es percibida por la mayoría de los sujetos como prácticas discriminatorias porque se consideran estas actitudes como aceptables y válidas, la respuesta es compartida, así como los prejuicios y valoraciones" (p. 64). A lo largo de la historia, la discriminación se ha convertido en un fenómeno social que afecta gravemente a la sociedad, es producto y genera desigualdades sociales en diferentes escenarios del entorno social, limitando los procesos de interacción entre los ciudadanos. Por tal razón, la discriminación conlleva a manifestaciones violentas que contribuyen con el rechazo y daños en la integridad de las personas afectadas.

Cuando se habla de discriminación, esta implica un juicio de valor, una opinión negativa que se tiene del otro, la cual lleva al rechazo y/o la exclusión. Teniendo en cuenta lo anterior, la discriminación se entiende como un fenómeno social y político que conlleva a hacer sentir en el otro inferioridad y diferenciación limitando los derechos, las libertades y las oportunidades de las personas en la sociedad (Cepeda, 2006).

En este orden de ideas, las personas que se identifican con una orientación sexual distinta corren un gran riesgo a ser víctimas de violencia física y psicológica por personas que no reconocen la diversidad de género, ya que están sometidas a estos procesos de discriminación, que reprimen y afectan los procesos de interacción social de las personas.

Ahora bien, si se habla de discriminación se entiende como un problema social, teniendo en cuenta que une un trato que evidentemente es distintivo, que aparta y es excluyente con un determinado grupo de personas. Esta discriminación conlleva el desarrollo de prácticas de rechazo, indiferencia e intolerancia hacia los demás, limitando el goce de los derechos y libertades fundamentales de los ciudadanos que las sufren, añadiendo que esto afecta la dignidad e integridad de los mismos. La discriminación no permite ser libre en la construcción de identidad y de elegir su libre desarrollo, no permite manifestar libremente sentimientos de amor, de deseo o simplemente de vestir, manifestarse, entre otros, lo que lleva a las personas a esconder sus preferencias y su identidad.

\section{En el ámbito universitario}

La investigación realizada propone revisar la situación en el ámbito universitario, en el cual hombres y mujeres, algunos aun construyendo su identidad, se relacionan en un mismo espacio. De este modo, se pretende analizar los grados o niveles de discriminación expresados en la radicalización de la exclusión, la aceptación de la diversidad, los conflictos por relación, o formas que se pueden observar por medio de rechazos, insultos e incluso la violencia física y emocional. Se entiende así que el ámbito universitario es el lugar territorial institucional que da forma a la universidad, espacio compartido por estudiantes, docentes, directivos, personal administrativo y demás, que conviven en el quehacer de la universidad y que tiene múltiples significantes para cada uno de los actores sociales que lo viven.

Se parte de la hipótesis desde la cual, el principal problema de las manifestaciones de discriminación que se evidencian en la institución Universitaria Antonio José Camacho tiene que ver con que los estudiantes no lo ven como un problema que afecte la intimidad e integridad de sus compañeros, lo ven como actos inofensivos mediante los cuales se divierten sin ocasionar daño alguno según su percepción. La concepción es que la interacción basada en discursos disruptivos, matoneo y prácticas de intimidación, exclusión y segregación de los estudiantes con orientación sexual diversa impiden el libre desarrollo de la personalidad de los estudiantes, promoviendo la inseguridad, el rechazo y la exclusión en contra de ellos. 
En ese sentido, la discriminación impide que se promuevan los procesos naturales de interacción entre los estudiantes limitando la libertad de ser y expresarse de cada uno de ellos; repercute en el entorno social atentando contra la integridad de las personas, afectando la naturaleza en los procesos de formación. Las manifestaciones de discriminación se relacionan con la búsqueda de oprimir y dominar al otro, en esto radican los estereotipos que se han impuesto social y culturalmente.

Ahora bien, la Institución Universitaria Antonio José Camacho y la universidad como institución en general presentan el gran desafío de trabajar en la diversidad. La universidad es por definición el espacio concreto que permite la pluralidad y multiculturalidad, en este orden ideas, ¿cómo se viene dando el proceso en la realidad práctica y cotidiana de los estudiantes?, ¿de qué forma se asume la diversidad y, en específico, la diversa orientación sexual por parte de los estudiantes de la jornada diurna en la Antonio José Camacho?

\section{Sobre la diversa orientación sexual, una aproximación a las investigaciones frente al tema}

Para la construcción de un estado del arte de acuerdo a las percepciones sociales y culturales sobre la discriminación hacia la diversidad sexual, es pertinente revisar el conjunto de investigaciones que se ordenan en este texto planteando las lógicas de discriminación sobre la diversidad sexual.

Autores como Samaniego y Bermúdez (2015, p.12) y Cornejo (2018, p.20) exponen que la discriminación que existe contra homosexuales en planteles educativos es alarmante, donde las principales agresiones se generan dentro de las aulas de clase, de forma verbal y física lo cual convierte los espacios educativos como un lugar inseguro y ajeno, a las orientaciones sexuales diferentes de la heterosexual, por lo cual los autores manifiestan que debe de ser abolido todo uso de lenguaje homofóbico o discriminatorio dentro de los salones de clases. La discriminación hacia la diversidad sexual en el ámbito universitario tiene que ver con la no aceptación de las personas que se identifican con orientaciones sexuales diferentes a la heterosexual. De acuerdo a List (2016), en las instituciones de educación superior no se han generado estrategias para atender a la comunidad vulnerada, es decir que no existen programas de socialización y cultura para evitar discriminación a personas con diferentes orientaciones sexuales y así disminuir la violencia de género y sexual (p. 2-6). Lo cual acrecienta la brecha de desigualdad y discriminación.

Por otra parte, Castedo y Tombesi (2019, Párr. 2-9), y Alventosa (2008), mencionan que en la actualidad las relaciones sostenidas con persona del mismo sexo, difiere mucho entre países, en algunos incluyen en sus constituciones la prohibición por discriminación por orientación sexual, tales como Sudáfrica, Bolivia, Ecuador, México, Nepal, Portugal, Suecia, Fiyi y Suiza, y en otros donde establecen la pena de muerte por sostener relaciones con personas de su mismo sexo, como Arabia saudita, Emiratos árabes, Irán y otros países de medio oriente, donde es inconcebible la diversidad sexual, sin embargo, De la Serna (2017, Párr. 1-3), sostiene que en el lado occidental, los individuos indiferentemente de sus orientaciones sexuales se pueden casar y adoptar hijos, como ocurre en gran parte de Europa, EE.UU y América Latina. No obstante, México presenta una cultura fuertemente conservadora lo cual la convierte en uno de los países con mayor afluencia de homófobos y generación de crímenes hacia las personas con orientación sexual diversa. De otro lado, Rodríguez y Toro (2002, p. 64-67) y Corsi (1995), presentan desde un punto de vista psicológico que los hombres han construido su identidad de género en oposición a la identidad femenina que por lo general se caracteriza a una mujer; de esta forma el hombre es lo opuesto a lo asignados a la mujer. A partir de esta situación se crean los mitos y creencias que se exteriorizan al caracterizar a un niño, en la construcción de su identidad masculina, pero el que un hombre desee a otro hombre no quiere decir que automáticamente va a actuar como mujer. Una persona del género masculino puede mantener los roles y estereotipos sociales asigna- 
dos a la masculinidad y modificar únicamente su objeto de deseo. Sin embargo, cuando un hombre se reconoce ante los demás como homosexual es encasillado, asumiendo que su comportamiento es opuesto a lo socialmente asignado. Toro-Alfonso, (2012), menciona que estos estigmas son considerados el desencadenante de factores que afectan a nivel personal y social a las personas con orientación sexual diversa, lo que de inmediato genera barreras y limitantes para los mismos, afectando de manera significativa su desarrollo y aprovechamiento pleno de lo que la sociedad ofrece para la comunidad en general, por el solo hecho de que su orientación sexual no es lo culturalmente aceptado (Jaramillo y Moreno, 2018. p. 10).

En otro orden de ideas, no todas las personas se sienten identificas de acuerdo con las opciones que las categorías de genero ofrecen. Según Rodríguez y Toro (2012), de ahí la necesidad de reconocer el papel que juega el cuerpo a la hora de expresar la forma de ser de una persona. Los autores expresan que los aspectos sociales influyen, trascienden y marcan de manera significativa el actuar de una persona, si se reconocen estos factores, la pregunta es ¿por qué las personas no traspasan las barreras paradigmáticas que continúan partiendo de nociones radicales? (p. 29).

Las investigaciones de Hernández \& Quintero (2009), Mogrovejo (2008), han centrado su atención en definir la diversidad en razón a la sexualidad, en su orientación, esta incluye al hombre y la mujer, y tiene que ver con la preferencia que estos sujetos tengan, es decir, que un hombre no necesariamente tiene que sentirse atraído por una mujer y que una mujer no necesariamente deba sentir gusto por un hombre. La orientación sexual determina en un ser humano una decisión que involucra no solo su cuerpo sino también sus emociones; una persona puede sentirse atraída por otra que sea de su mismo sexo y puede llegar a ser correspondida de igual forma.

Otro aspecto de las investigaciones como las de Barrientos \& Cárdenas (2013), De Jesús (2000), Se- villano (2015), Buendía, R y Douglas, O (2012), se ha centrado en los derechos de las personas que hacen parte de la diversidad sexual. La literatura resalta que por más luchas que han librado no han logrado tener derechos igualitarios a los del resto de la población, esto debido a la falta de interés de los estados, la estigmatización y la discriminación que tiene la sociedad frente al tema. No se tiene garantías en cuanto al trabajo, ya que no existe una ley que le impida a un empresario despedir a una persona por no estar de acuerdo con su orientación sexual, y a pesar de que estamos en una sociedad moderna y los tipos de familia han cambiado con el paso de los años, aun se ven estigmatizadas las familias cuyos padres son del mismo sexo, lo que significa que en cuanto a legislación tampoco existen garantías.

Respecto a los tipos de discriminación, estos surgen a partir de los estereotipos que se han impuesto socialmente, con ello se ha enseñado como debe ser el modelo y conducta de una persona para que sea aceptada dentro de un grupo o comunidad. La ausencia de reconocimiento de este fenómeno es otro aspecto muy arraigado en la conciencia, así como la actitud patriarcal a escala social. La discriminación ha llevado también a situaciones como suicidio, depresión, alcoholismo y/o drogadicción. Los derechos humanos se han creado y promovido con el fin de que la humanidad se respete movilizándose a favor de ellos para lograr vivir con dignidad, en tanto principio superior que ningún ordenamiento jurídico puede desconocer (Carpizo, 2011).

\section{Causas de la discriminación a la diversidad sexual}

Las principales causas de la discriminación hacia la población sexualmente diversa tienen que ver con las construcciones sociales y el sostenimiento de una cultura patriarcal, las concepciones culturales que privilegian a la heterosexualidad como la única forma de expresión de la sexualidad, la definición de la homosexualidad como anormalidad, la concepción de familia tradicional y las creencias religiosas que desconocen la complejidad humana. 
En el marco social este tipo de discriminación se ha venido presentando con mayor fuerza en los últimos años, esto se debe a los estereotipos que la sociedad nos ha impuesto durante generaciones. Autores como Galán \& Ignacio (2009), Briceño (2011), Castelar y Briceño (2017), Rosales (2016) y Mogrovejo (2008) plantean que la única orientación sexual que es entendida y aceptada en el contexto social es la heterosexualidad. A través de la cultura se han transmitido formas de sentir y pensar en la gran mayoría de la sociedad; ahora bien, teniendo en cuenta que el ser humano construye su identidad a partir de ello, las formas culturales establecidas también determinan los prejuicios y estigmas en cada uno de los individuos. Con ello se ha obligado involuntariamente al individuo a interiorizar que la atracción por el mismo sexo es algo que es inmoral, porque no es socialmente aceptada y afecta el orden natural, lo cual se puede observar en el reconocimiento de la familia tradicional, en la heteronormatividad, en la discusión teológica y biológica de la división de los roles para hombre y mujer.

El rechazo inicia con comentarios y burlas que al parecer son inofensivas, pero a lo largo del proceso van tomando mayor fuerza llegando a límites como el acoso, la violación y el asesinato. Por otro lado, es importante resaltar que la falta de denuncias y la circulación de estadísticas oficiales permiten reconocer que se ha convertido en un conflicto social que está acabando con la dignidad y la vida de muchas personas. Es una lucha que no solo se está presente en el ámbito universitario, afecta todos los escenarios sociales (Inter-American Commission on Human Rights, 2015).

\section{Hacia una comprensión de las manifestaciones de discriminación}

El fenómeno social que se aborda trata de la discriminación a la diversidad sexual en el ámbito universitario, teniendo como fuente de referencia lo que se expone en la teoría queer, que define a las diferenciaciones por orientación sexual como un producto social y no natural, que critica la heterosexualidad como única forma de definición de la sexualidad, pero que, además, define y hace críticas a las nociones de género y sexualidad.

La teoría queer (considerada como la teoría torcida) inicia como un movimiento en EE.UU., que entiende al ser humano como único y diverso, es decir, que la diversidad está enmarcada en la humanidad, por ende, natural, haciendo una crítica al sistema social que asume la diversidad como división, como producto social y cultural. Entiende la teoría que la discriminación es también producto social y cultural, establecido por la generación de una concepción de superioridad de una etiqueta social sobre la otra, utilizando prácticas que determinan el uso de la violencia como una forma de rechazo (Fonseca Hernández y Quintero, 2009).

Soto (2009) señala que "la declaración de la homosexualidad perturba la integridad y los fundamentos del orden social, con lo cual la represión del discurso homosexual garantiza la sociabilidad mientras esta permanezca en silencio". Según lo mencionado por este autor, se puede ratificar que la diversidad sexual es reprochable siempre y cuando se manifieste públicamente, su permanencia en el anonimato genera la aprobación de la heteronormatividad, aun latente en nuestra sociedad, que siempre está condenando la relación entre personas del mismo sexo, no por el hecho de tener la relación, sino por hacer manifestaciones expresando sus gustos y preferencias sexuales frente a los demás. En otros términos, lo que realmente importa es mantener lo ya establecido socialmente, así sea con base en apariencias, ya que eso es lo que se ha impuesto a lo largo de la historia.

Según Fonseca (2009), desear a un hombre no implica necesariamente identificarse como mujer, y desear a una mujer no involucra una identificación masculina; el sistema heterosexual es una lógica imaginaria que continuamente reproduce su propia ingobernabilidad. La naturalización de la heterosexualidad no es más que un espejismo (Soto, 2009). En tal sentido, el sexo biológico y la orientación sexual no están vinculadas una a la otra, debi- 
do a que la orientación sexual nace a partir de una construcción sociocultural.

Por su parte, la presente investigación analiza en el ámbito universitario las relaciones y cómo estas pueden afectar la dinámica entre estudiantes. Es así como el interaccionismo simbólico trata de comprender a las sociedades por medio de la comunicación que se manifiesta a través de símbolos y significados, y desde ellos, el desempeño comportamental frente a lo que se significa, lo cual constituye la clave para entender el juego de intersubjetividades. Las personas actúan según la interpretación que tengan de su entorno y construyen su identidad a partir de las relaciones interpersonales que surgen socialmente, aquí es importante la configuración y construcción territorial en el espacio habitado, pues como lo refiere Mafessolli (2004), espacio y territorio están estrechamente ligados en tanto dan sentido y apropiación del lugar habitado o vivido. Desde la sociología el interaccionismo simbólico trata de ver la acción individual de las personas como el resultado de un sistema normativo general, en cual influye la cultura en que se construye el individuo (Blúmer, 1982).

Lo mencionado anteriormente por Blúmer (1982) hace referencia a la comunicación como la base fundamental para la construcción de una sociedad. Sin interacciones simbólicas los seres humanos no podrían conformar su identidad personal, en este caso, frente a la discriminación a la diversidad sexual, es necesario reconocer que puede tratarse de un tema que puede incluir la exclusión $y$ rechazo de un individuo hacia otro como manifestaciones de la concepción individual. Ahora bien, el espacio y territorio de interacción también desarrolla nociones de comportamiento; para este caso puntual, el ámbito universitario es el espacio territorio que crea distintas configuraciones entre lo simbólico, lo apropiado y lo vivido, es decir, que el comportamiento en este escenario para un mismo actor social puede ser distinto a lo vivenciado en un espacio territorio diferente, tal como lo es el barrio, la comuna o, incluso, la escuela.
De otro lado, las manifestaciones de discriminación no solo se involucran en el marco étnico-racial, la discriminación va más allá debido a que se cuestionan los pensamientos e ideologías de las personas que son diferentes al resto de lo que la sociedad considera como normal o a lo que están acostumbradas. Las manifestaciones de discriminación no solo involucran esos actos de rechazo e indiferencia hacia el otro, también se refleja en dos características fundamentales: la injusticia, ya que estamos sometidos a un sistema de dominación, y la opresión, la cual se manifiesta por estereotipos, estigmas y prejuicios que se han establecido socialmente (Alarcón, B y Toquica, A., 2016). En ese sentido, se entiende las manifestaciones de discriminación como las actitudes, comportamientos, acciones de repulsión, de exclusión que surgen en la dinámica de interacción simbólica entre sujetos sociales y que construyen y contribuyen a la opresión, la desigualdad y la injusticia social.

\section{METODOLOGÍA}

La investigación permitió acercarse al fenómeno para deducir que el espacio físico es relevante en términos de las acciones que determinan la discriminación y que, aunque se ha avanzado en la generación de condiciones para la inclusión efectiva, los procesos sociales, las relaciones y prácticas al interior de las instituciones, del contexto social, no es aún una fuente garante para este proceso. La metodología empleada fue un estudio de tipo comprensivo-explicativo desde su profundidad y por temporalidad de tipo sincrónico, utilizando la combinación de métodos desde la complementariedad para, de esta manera, comprender y explicar las manifestaciones de discriminación existentes entre los estudiantes de la sede norte jornada diurna de la Institución Universitaria Antonio José Camacho durante el periodo académico 20191. La investigación se desarrolló desde un enfoque analítico y hermenéutico, haciendo uso de instrumentos cuantitativos como la encuesta e instrumentos cualitativos como entrevistas semiestructuradas, observación no participante y un grupo 
de discusión para la recolección de la información. El análisis de la información se desarrolla con la triangulación de datos desde una perspectiva de complementariedad.
Se desarrolló un muestreo aleatorio simple a partir de la identificación de la totalidad de estudiantes de la jornada de la mañana en términos del desarrollo de la encuesta.

Tabla 1. Datos Población de estudio

\begin{tabular}{llllc}
\hline \multicolumn{1}{c}{ Ubicación } & Población & Tamaño de la muestra & Margen de error & Nivel de confianza \\
\hline $\begin{array}{l}\text { Sede Norte- Jornada } \\
\text { diurna }\end{array}$ & 512 & 126 & $\mathbf{1 0} \%$ & $\mathbf{9 9 \%}$ \\
\hline
\end{tabular}

Fuente: Elaboración propia de los autores

Para obtención del tamaño de la muestra, margen de error y nivel de confiabilidad se utilizó la fórmula de ecuación estadística para proporciones poblacionales.

Para las técnicas cualitativas, las entrevistas se realizaron a estudiantes que se reconocen como sexualmente diversos, se desarrolló un grupo de discusión con estudiantes que mostraban tener mayores resistencias a conversar sobre el tema (identificados a partir del desarrollo de la encuesta) y finalmente, se realizaron observaciones a diferentes espacios comunes que los estudiantes comparten, tales como la cafetería, los baños, el aula, entre otros. A continuación, el artículo analiza algunos datos cuantitativos y cualitativos del proceso de investigación.

\section{Manifestaciones de discriminación en la Uniajc}

Las características fundamentales de la población que acompañó el estudio son los estudiantes de la jornada de la mañana de la sede principal de la Institución Universitaria Antonio José Camacho; en ella, los estudiantes de programas como Ingenierías y Trabajo Social comparten en su mayoría el ser de y residir en la ciudad de Cali (81\%) y sus alrededores. En términos socio económicos pertenecen a los estratos: 2 (42\%), 3 (25\%), 1(20\%) y 4 (11\%). El 51\% se reconocen en el género masculino y el $49 \%$ en el femenino. Por otra parte, el $90 \%$ son heterosexuales, el $5 \%$ son bisexuales y el $4 \%$ son homosexuales. El rango de edad más habitual entre los estudiantes es de 19 a 25 años con un $53 \%$.
El $60 \%$ se reconocen como católicos siendo así el porcentaje más alto, el $20 \%$ como cristianos, el $1 \%$ como testigos de Jehová y el 18\% en otra religión no especificando cuál. De otro lado, el $39 \%$ de los estudiantes se reconocen como mestizos, el $34 \%$ como negro/afrodescendiente, el 1\% como indígenas y el $25 \%$ no se reconoce dentro de ninguna etnia. Datos que se ilustran en las figuras 1 y 2.

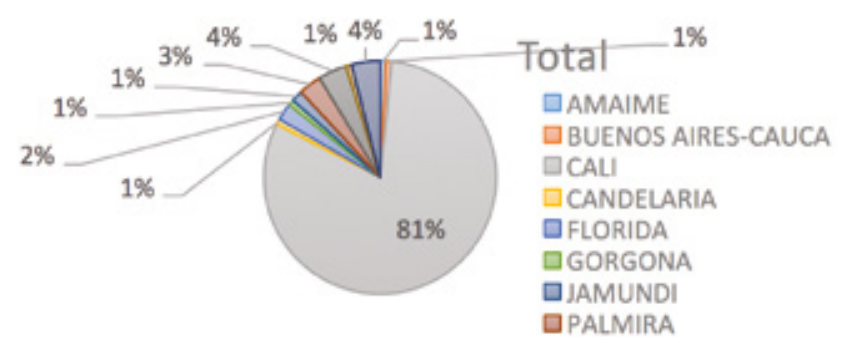

Figura 1. Lugar de Residencia. Fuente Elaboración propia.

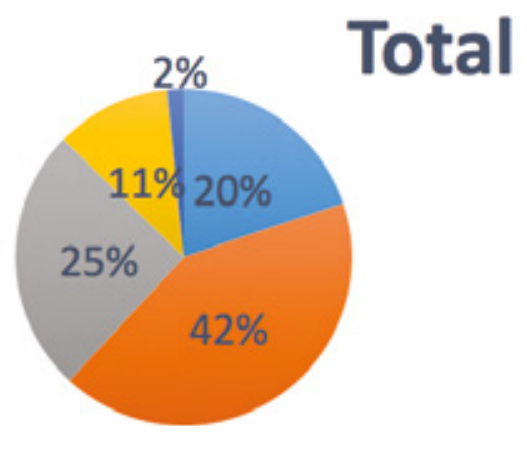

Figura 2. Estrato socio económico. Fuente Elaboración propia. 
Los datos poblacionales permiten inferir en general unas características homogéneas, a partir de las cuales se presenta un reconocimiento personal de condición diversa. Las mayores diferencias de la población hacen referencia a las características étnicas.

En cuanto a lo que los estudiantes manifiestan frente a la diversidad, existe una tendencia a definir que al interior de la universidad se perciben casos de discriminación en un $61 \%$, de los cuales un $14 \%$ percibe que la discriminación se realiza en contra de las personas con orientación sexual diversa, como se muestra en las figuras 3 y 4 .

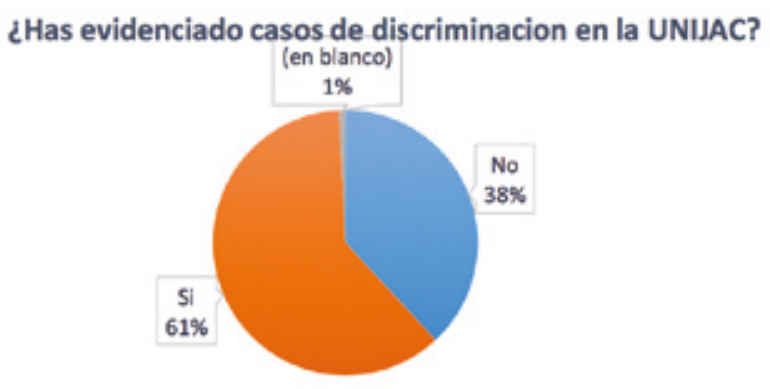

Figura 3. Percepción sobre casos de discriminación. Fuente: Elaboración propia.

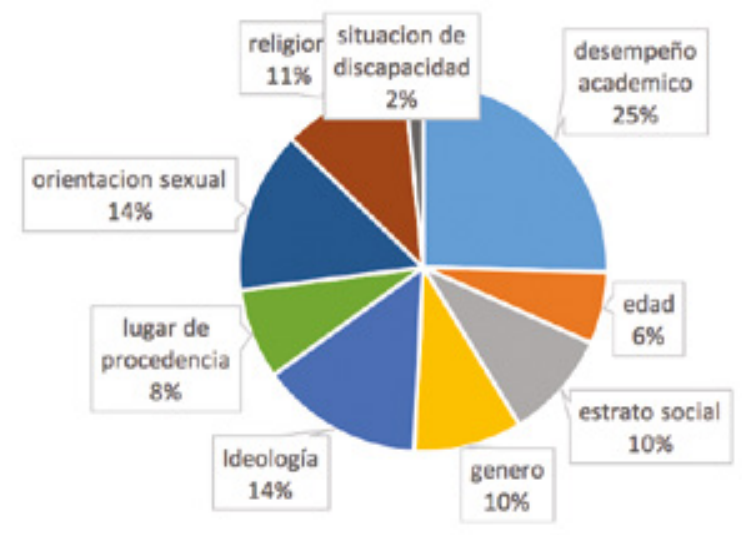

Figura 4. Percepción de casos de discriminación. Fuente: Elaboración propia.
Relacionando estos resultados con la pregunta: ¿alguna vez se había sentido discriminado dentro de la universidad? Tal como se muestra en la figura 5, el porcentaje fue menor. Del $100 \%$ de los encuestados, el $26 \%$ manifestó que en algún momento sí llegó a sentirse discriminado, pero el tipo de discriminación que más habían percibido era por orientación sexual, seguida por ideología y desempeño académico.

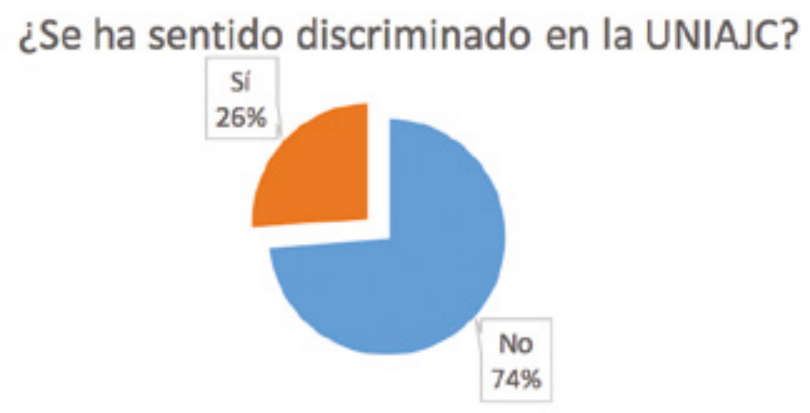

Figura 5. Sentido de la discriminación. Fuente: Elaboración propia.

$\mathrm{Al}$ respecto, es posible concluir en este momento que, aunque los datos no presenten la discriminación en términos de mayorías porcentuales, existe la percepción sobre la existencia de discriminación, siendo significativa en esta percepción la desarrollada en contra de quien presenta orientación sexual diversa. El hecho de sentirse discriminado supera el nivel cuantitativo de los datos y perfila la existencia de un fenómeno que, tal como se ha manifestado anteriormente, las mayorías no visualizan por haberse naturalizado.

La encuesta realizada con el fin de identificar las manifestaciones de discriminación pudo establecer los siguientes resultados. En primer lugar, las manifestaciones de discriminación no parecen visualizarse como recurrentes ni cotidianas, tan solo un $2 \%$ contestó que le da risa ver a una persona con orientación sexual diversa; un 23\% manifiesta hacer chistes a compañeros heterosexuales haciendo referencia a características homosexuales ( $\mathrm{Fi}$ gura 6); a su vez, se pudo verificar el sentimiento de repulsión por parte de los estudiantes a conduc- 
tas de amor públicas entre homosexuales hombres y mujeres, tal como lo demuestran las figuras 7 y 8 .

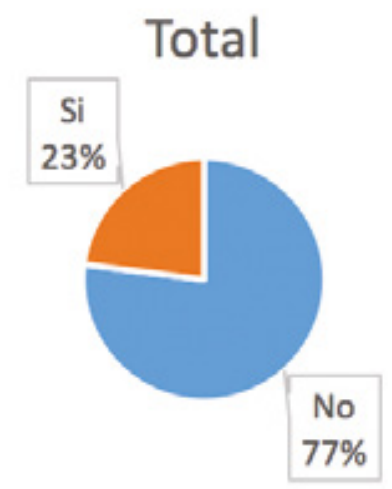

Figura 6. ¿Hace chistes a sus compañeros de clase heterosexuales haciendo referencia a características homosexuales? Fuente: Elaboración propia.

\section{¿Qué tan de acuerdo está en que una pareja homosexual se bese en público?}

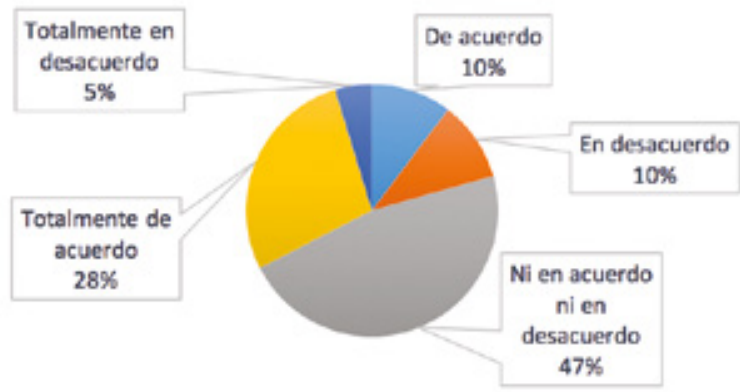

Figura 7. Aceptación o no de conducta pública. Fuente: Elaboración propia.

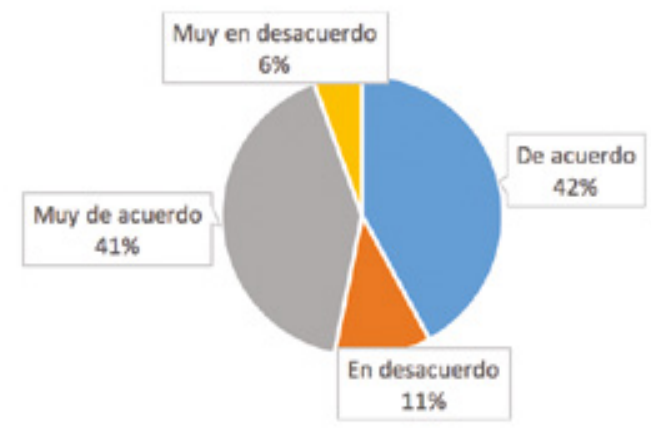

Figura 8. ¿Respeta la diversidad sexual siempre y cuando no se exhiban en público? Fuente: Elaboración propia.
A partir de lo anterior se puede inferir que la discriminación hacia la conducta pública referente a expresiones de amor como tomarse de la mano o de interactuar con libertad frente a su orientación, indican una aceptación condicionada que mantiene los estereotipos aduciendo una concepción de tolerancia mediada por la actuación pública.

Por otra parte, se pueden observar acciones de discriminación desde la interacción, el discurso y los lineamientos heteronormativos. Por ejemplo, las figuras 9 y 10 muestran cómo los encuestados asumen posiciones religiosas de discriminación frente a la conducta pública, de burla y no consentimiento de las relaciones homosexuales, además de sexista y violenta, como se puede ver en la Figura 10, que ubica la relación entre mujeres como fetiche masculino y posibles acciones violentas.

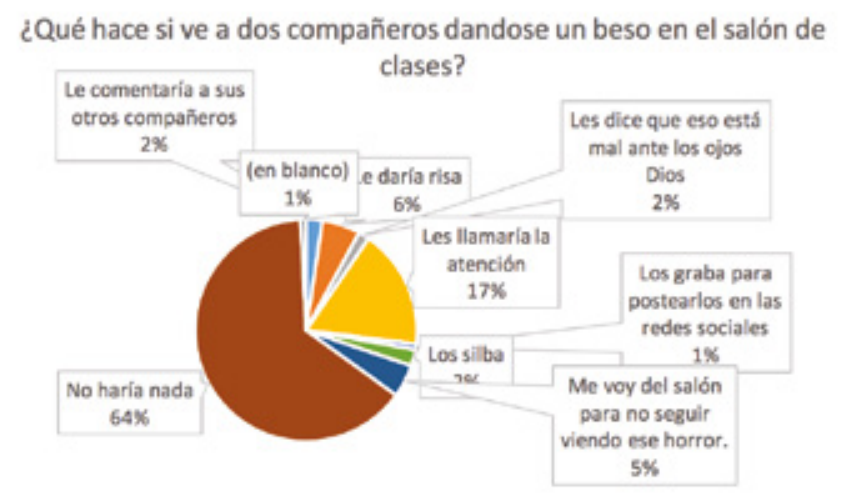

Figura 9. Acciones frente a la conducta pública. Fuente: Elaboración propia.

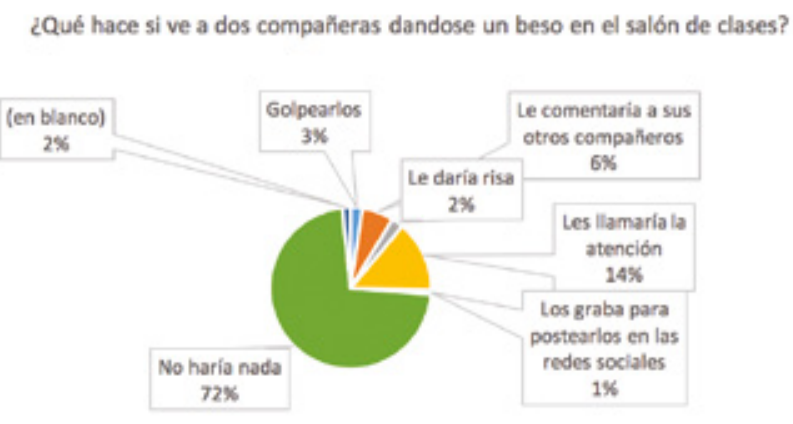

Figura 10. Acciones sobre la conducta pública en mujeres. Fuente: Elaboración propia 
Finalmente, se percibe que la conducta frente a la relación con la diversidad puede pasar a la violencia física si se observa un acercamiento entre personas del mismo sexo entre los estudiantes, lo que evidencia que es una tolerancia frente a la diversidad que está mediada por los límites en la interacción social (Figura 11).

\section{Total}

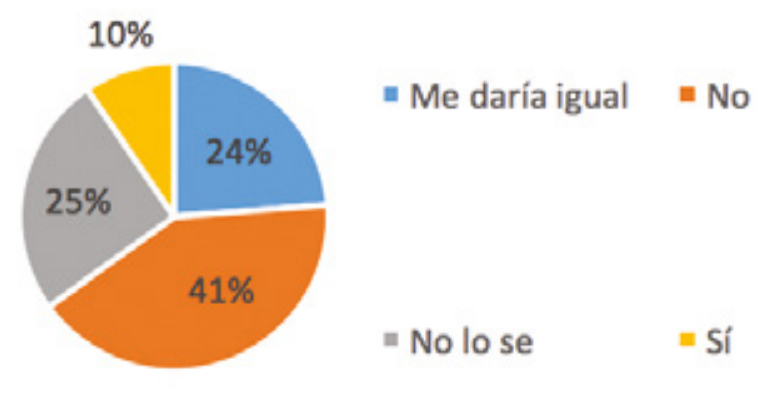

Figura 11. Reacción frente a la insinuación por alguien del mismo sexo. Fuente: Elaboración propia.

Con respecto a los datos cualitativos, los estudiantes entrevistados manifestaron rechazo o discriminación por su orientación. Este tipo de manifestaciones discriminatorias, según los instrumentos aplicados, se ha evidenciado más hacia los hombres homosexuales; en ellos es donde más se genera el rechazo hacia su forma de ser y expresarse como tal; en las mujeres es más visto como algo erótico o como una forma de generar excitación para quienes presencian los actos amorosos.

Sin embargo, para uno de los entrevistados, las manifestaciones de discriminación no son tan fuertes en la universidad como lo son en otros espacios:

En la universidad no lo he sentido, de hecho, mis compañeras me acosan, jejeje, nunca he sentido como que digan no lo voy abrazar porque es gay, no. Dentro de la universidad no, nunca. La verdad no he evidenciado ni dentro ni fuera del salón de clases. Entrevista No. 3

Los entrevistados cuentan acerca de las experiencias duras que tuvieron que sufrir en el colegio debido al bullying por parte de sus compañeros, quienes rechazaban sus actitudes "amaneradas", un comportamiento que no fue aceptado y causó dificultades en los procesos de formación académica y desarrollo de estas personas. Esto permite deducir que la universidad ha sido un escenario que ha permitido otro tipo de relaciones, más abiertas y no tan discriminatorias.

\section{CONCLUSIONES Y RECOMENDACIONES}

La universidad es un escenario o ámbito en el cual la diversidad es más aceptada y no tan discriminada. Sin embargo, las manifestaciones de discriminación ante personas siguen siendo un fenómeno que se genera en los diálogos, discursos, bromas, silbidos y en los imaginarios que se tienen sobre las personas con orientación sexual diversa.

Las percepciones de los estudiantes encuestados frente a la conducta pública de las personas con orientación sexual diversa constituyen las principales manifestaciones en donde se percibe la homofobia, ya que en cada una de sus opiniones se manifestaban discursos dirigidos a la inconformidad frente a la conducta sexual y, del mismo modo, se pudo caracterizar que las formas de exclusión se dan por los comportamientos en público de las personas con orientación sexual diversa. Se observa en las construcciones simbólicas la acción basada en lo moralmente aceptado y también en la tendencia a encasillar estas personas en unas etiquetas que generan formas de agresión. También se interpretó que el discurso de los estudiantes frente a la construcción cultural de género se sigue rigiendo en su mayoría por la heteronormatividad, donde el género masculino solo es reconocido en el hombre y el género femenino en la mujer, desconociendo así otros tipos de definición de género.

No hay un reconocimiento tácito de la intolerancia por parte de los estudiantes, pero en el discurso se puede observar que la tolerancia y el respeto se limita a que la persona diversa no realice prácticas que corresponden a sus ejercicios de libertad. En 
síntesis, la presión social sobre el comportamiento público sigue siendo un fenómeno que no se ha trabajado.

Otro factor clave que se pudo identificar es la apropiación del espacio. Siendo la universidad un escenario más tolerante para el estudiante diverso, se observa con la investigación que en otros contextos los mismos estudiantes asumen más discriminación que en el contexto universitario.

La discriminación hacia las personas que se reconocen como diversas sexualmente debe ser atendida por medio de políticas públicas e institucionales que se enfoquen en promover el multiculturalismo, la aceptación y el respeto cotidiano a la diversidad. La universidad sigue siendo un espacio libre o así percibido, sin embargo, la sociedad sigue discriminando de manera tal que afecta la libertad individual y los derechos de las personas. Los estudiantes con diversidad sexual distinguen que el ámbito universitario les permite ser, pero la sociedad no.

\section{REFERENCIAS BIBLIOGRÁFICAS}

Alarcón, B, y Toquica, A. (2017). Discriminación y violencia en jóvenes universitarios de Bogotá, 2016 (Tesis de maestría). Universidad Santo Tomás, Bogotá, Colombia.

Alcaldía Mayor de Bogotá (2007). Diversidad sexual en la escuela: dinámicas pedagógicas para enfrentar la homofobia. Bogotá sin indiferencia. Recuperado de http://repositoriosed.educacionbogota.edu.co/ handle/001/1117

Cepeda, (2012). Discriminación. Tomado de: http://discriminacionproblema.blogspot.com.co/

Barrientos, J., \& Cárdenas, M. (2013). Homofobia y calidad de vida de gay y lesbianas: una mirada psicosocial. Psykhe (Santiago), 22(1), 3-14
Buendía, R. y Douglas, O. (2012). Matrimonio entre personas del mismo sexo en Chile: hacia una construcción jurisprudencial nacional a partir de los derechos humanos. Disponible en http://repositorio. uchile.cl/handle/2250/113870

Blúmer, H. (1982). La posición metodológica del interaccionismo simbólico. El interaccionismo simbólico. Perspectiva y método, 1-44.

Briceño, Mónica. (2011). Reconociendo la diversidad sexual: Representaciones sociales acerca de diversidad sexual en profesionales de Trabajo Social. Trabajo de grado para optar al título de Trabajadora Social. Universidad del Valle, Cali.

Castelar, A. y Briceño, M. (2016). “¿Qué dirá Dios?”: Religiosidad y prejuicio en las representaciones de la diversidad sexual de trabajadoras sociales en Cali. La Manzana de la discordia, 9(1), 103-115.

Carpizo, J. (2011). Los derechos humanos: naturaleza, denominación y características. Cuestiones constitucionales, (25), 3-29

Galaz, C., Troncoso, L., \& Morrison, R. (2016). Miradas críticas sobre la intervención educativa en diversidad sexual. Revista latinoamericana de educación inclusiva, 10(2), 93-111.

Galán, P., \& Ignacio, J. (2009). Entender la diversidad familiar. Barcelona: Ediciones Bellaterra.

De Jesús, M. (2000). Sin licencia para amar: Prohibición de adopción a personas y parejas homosexuales y lesbianas en Puerto Rico. Rev. Jur. UPR, 69, 12811341.

Fonseca Hernández, C., \& Quintero Soto, M. L. (2009). La Teoría Queer: la de-construcción de las sexualidades periféricas. Sociológica (México), 24(69), 4360 .

Jaramillo Gómez, I., y Moreno Méndez, K. J. (2018). Percepciones de estudiantes gays y lesbianas sobre diversidad sexual en contexto universitario (Tesis de 
pregrado). Pontificia Universidad Javeriana, Cali, Colombia.

Maffesoli, M. (2004) “La especialización del tiempo", en Ciudades 61. Puebla: RNIU.18.

Mogrovejo, N. (2008). Diversidad sexual, un concepto problemático. Trabajo social UNAM, (18), 62-71.

Sevilla -González, M. (2007). El discurso homofóbico como expresión de la discriminación sexual. En G. Soberón, \& D. Feinholz (Eds.). Homofobia y Salud (pp. 63-76). México, DF: Secretaría de Salud.

Sevillano Angulo, G. A. (2015). Necesidad de regular dentro de la legislación laboral ecuatoriana el meca- nismo legal que permita el ejercicio pleno de los derechos laborales de las personas sexualmente diversas (glbti), de conformidad con lo que dispone el artículo 1 numeral 2 de la Constitución de la República del Ecuador (Tesis de pregrado). Universidad Nacional de Loja, Ecuador.

Rosales G. (2016). Heteronormatividad y heterosexualidad obligatoria. Recuperado de http://rupturacolectiva.com/heteronormatividad-y-heterosexualidad-obligatoria/

\section{AUTORES}

Carlos Alberto Sarria Trejos: Magister en Política Pública y Trabajador Social de la Universidad del Valle, Docente Investigador Universitario de la Institución Universitaria Antonio José Camacho, programa Académico de Trabajo Social y Director de la Especialización en Ciudadanía, Derechos y Gestión de Paz. Correo electrónico: casarria@admon.uniajc.edu.co.

Lizeth Daniela García Chico: Trabajadora Social y estudiante de la Especialización en Ciudadanía, Derechos y Gestión de Paz en la Institución Universitaria Antonio José Camacho. Correo electrónico: daniela_garcia_14@hotmail.com
Dorlaomer Villa Guzmán: Trabajador Social de la Institución Universitaria Antonio José Camacho. Correo electrónico: dvillag@admon.uniajc.edu.co.

Vivian Figueroa Mejía: Trabajadora Social de la Institución Universitaria Antonio José Camacho. Correo electrónico: vfigueroa@admon.uniajc.edu.co. 